\title{
SUBGLOTTIC HAEMANGIOMA: TWO INFANTS WITH LARYNGEAL STRIDOR
}

BY

\author{
C. A. HOLBOROW \\ From The Hospital for Sick Children, Great Ormond Street, London
}

(RECEIVED FOR PUBLICATION SEPTEMBER 24, 1957)

The literature contains records of 18 infants with stridor due to haemangiomata in or just below the larynx. Two of these were reported by Mr. James Crooks in this Journal (Crooks, 1954). Since then he has had three further cases at The Hospital for Sick Children, Great Ormond Street, and I am indebted to him for permission to publish details of two of them, bringing the total number of cases recorded up to 21 .

\section{Case Histories}

Case 1. (Fig. 1). C.E. was a girl born on January 6, 1956. Laryngeal stridor developed after an attack of acute

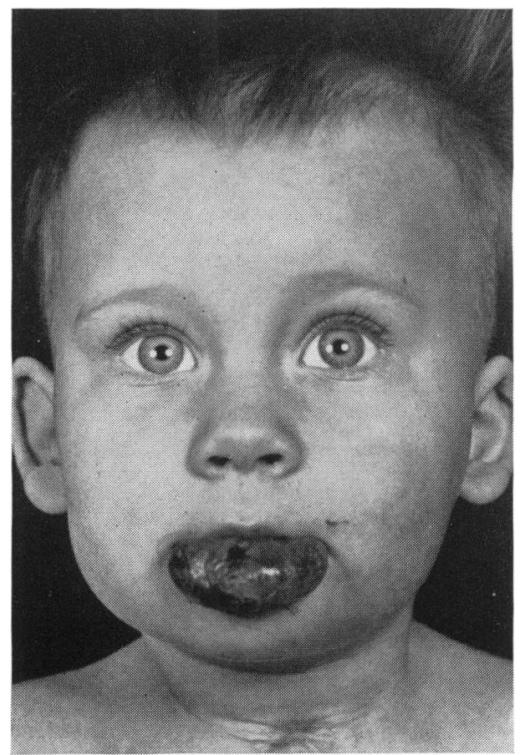

FIG. 1.-Case 1. Child after removal of tracheostomy tube.

bronchitis at 6 weeks of age. The stridor continued with intermittent pyrexia, and a laryngoscopy was performed at another hospital when she was 3 months old. The larynx was found to be very red; no web or tumour was seen. The stridor continued despite anti- biotics and a month later she was transferred to The Hospital for Sick Children. On examination there was biphasic stridor with rib and suprasternal recession. The child had a good colour and was not distressed. The only other abnormality was a haemangioma of the lower lip. A laryngoscopy was performed under a general anaesthetic. The larynx was red and some subglottic swelling was seen which was thought might be due to infective oedema. Following this examination the stridor was very marked but was relieved by nursing in an atmosphere saturated with water vapour. A respiratory infection delayed further investigation for two months when a further laryngoscopy showed a symmetrical subglottic swelling which appeared to be either

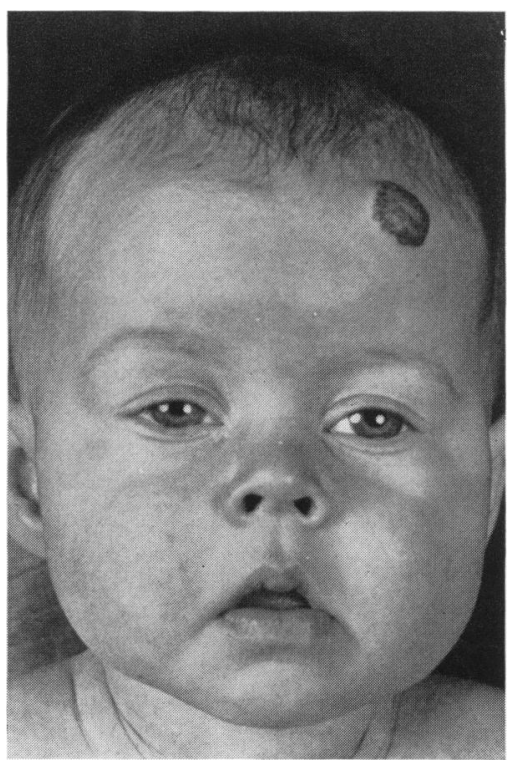

FIG. 2.-Case 2.

inflammatory oedema or a haemangioma. The stridor and respiratory obstruction which followed this examination necessitated a tracheostomy.

Because of the haemangioma on the lip it was assumed that the swelling seen was haemangiomatous and the 
subglottic region was irradiated by means of deep x-ray (250 R.). The treatment was followed by considerable tracheal obstruction and respiratory distress. A month later laryngoscopy showed that the subglottic swelling was larger, and a biopsy confirmed the diagnosis of haemangioma. A second course of irradiation (200 R.) caused no reaction and one month later an attempt was made to remove the tracheostomy tube under sedation. The child tolerated laryngeal respiration for two days until increasing dyspnoea and stridor compelled the replacement of the tube. Bronchospasm and respiratory infection followed and congestive cardiac failure developed. This was controlled with digoxin and antibiotics and a third course of deep x-ray was then given. The treatment provoked no reaction but the tube was left in place because of a pyrexia due to teething and it was finally removed under sedation seven months after admission. Progress was satisfactory and a later laryngoscopy showed a good airway with only some residual swelling in the subglottic region. The child was discharged home eight months after admission. She has attended for treatment to her lip but has had no more stridor.

Case 2. (Fig. 2.) J.G., also a girl and a second child, was born on February 5, 1957. A laryngeal stridor was first noticed at 6 weeks of age and gradually became worse finally becoming very marked with a cold. There was some feeding difficulty. When seen at another hospital a lateral radiograph of the soft tissues of the neck showed a rounded swelling partly blocking the trachea below the cords. She was transferred to The Hospital for Sick Children and was found to have a biphasic stridor with rib recession. There was, in addition, a small haemangioma of the forehead which, the mother said, had gradually enlarged since birth. Laryngoscopy revealed a rounded swelling arising from the posterior wall of the subglottic region. A biopsy taken from this swelling showed that it was a partly canalized haemangioma. Considerable stridor followed this operation but a tracheostomy was avoided by nursing in a humid atmosphere for 24 hours. The subglottic region was irradiated twice with an interval of two weeks by means of deep x-ray ( $250 \mathrm{R}$. and 200 R.). No reactions followed and two days after the second treatment the stridor ceased. A month after admission she was discharged home and has had no subsequent trouble.

\section{Comment}

Haemangiomata are malformations rather than neoplasms and canalization accounts for their seeming growth (Willis, 1948). This gradual enlargement was observed in the first case recorded here. The condition is probably not as rare as the few records suggest and reports of a number of cases have appeared recently (Baker and Pennington, 1956). The records are all very similar. Though it has been suggested that this condition occurs more often in males there is no difference in the incidence between the sexes in the small number recorded. Stridor usually becomes apparent during the first three months of life as canalization occurs. It is the presenting symptom. The site of the haemangioma is usually the subglottic region or the upper trachea, though the supraglottic region has been affected and in one case (Baker and Pennington, 1956) the mediastinum and bronchi were involved. In over half the number there were other haemangiomata, usually on the head and neck. The mortality is considerable and nine of the 21 cases have died of respiratory obstruction.

The most satisfactory treatment has, in all cases, proved to be radiotherapy (Suehs and Herbut, 1940; Ferguson, 1944; Kasabach and Donlan, 1945; Crooks, 1954) and though there is some theoretical danger to the developing larynx there are no reports of such damage. The second case described here is the only one recorded where a proven haemangioma has been successfully treated without tracheostomy. The two case histories are an interesting comparison in this respect as the first shows that the hazards of a tracheostomy and the difficulty in removing the tube markedly increase the risk to the child and the time spent in hospital. In many cases, of course, tracheostomy must be performed on admission and an increase in the size of the haemangioma following irradiation may lead to sudden respiratory obstruction. However, provided that everything is in readiness for an emergency operation, the risk of irradiation without an elective tracheostomy appears to be justified.

\section{REFERENCES}

Baker, D. C. and Pennington, C. L. (1956). Laryngoscope (St. Louis), 66, 696.

Crooks, J. (1954). Arch. Dis. Childh., 29, 12.

Ferguson, G. B. (1944). Arch. Otolaryng. (Chicago), 40, 189.

Kasabach, H. H. and Donlan, C. P. (1945). J. Pediat., 26, 374.

Suehs, O. W. and Herbut, P. A. (1940). Arch. Otolaryng. (Chicago), 32, 783.

Willis, R. A. (1948). The Pathology of Tumours. London. 